\title{
Penser la catégorisation des formes sociales de production agricole en Europe centrale
}

\author{
Marie-Claude Maurel* \\ EHESS, CERCEC, bureau n ${ }^{\circ}$ 12, 105 boulevard Raspail, 75006 Paris, France
}

\begin{abstract}
Résumé - Dans les pays d'Europe centrale qui ont connu la collectivisation, l'agriculture se caractérise par des formes sociales inédites. Les structures des exploitations agricoles peuvent être identifiées suivant les catégories statistiques et normatives usuelles. L'article identifie les diverses formes sociales en considérant leur origine et leur évolution à la suite du processus de privatisation. La plupart de ces formes sociales, telles les petites exploitations de semi-subsistance ou les grandes exploitations sociétaires, diffèrent des formes existantes dans les anciens États membres de l'Union européenne. Fondé sur la catégorie normative de l'exploitation familiale, le «modèle agricole européen "se révèle inapproprié au vu de pays où les exploitations familiales moyennes et grandes n'existent presque pas. L'article examine les effets distributifs pervers de l'application d'une politique de soutien (le régime simplifié de paiements directs) à des structures d'exploitations pour lesquelles elle n'a pas été conçue.
\end{abstract}

Mots clés : structure agraire / petite exploitation / grande exploitation / agriculture collective / privatisation

Abstract - Thinking about the categorization of social forms in Central Europe. Farming is organized in different forms in the countries of Central Europe where collectivization had been implemented. The very different agrarian structures may be defined using conventional statistical and normative classifications. This article identifies the various social forms considering how they have arisen and evolved from the privatization process. Most of these forms such as the tiny semi-subsistence farms or the large corporate units are different than seen in the former member states of the European Union. Based on the assumption of a family norm, the "European model of agriculture" appears to be unsuitable to countries where large and medium family farms scarcely existed. The article considers the perverse distributive effects of extending an agricultural support policy (the so-called SAPS) to structures for which it had not been designed.

Keywords: agrarian structure / small farms / large farms / collective farming / privatization

\section{Introduction}

En Europe centrale, la recomposition des structures d'exploitations agricoles résulte de la rupture avec le collectivisme agraire. Si les classifications statistiques d'Eurostat permettent d'identifier les singularités structurelles de ces agricultures, elles sont impropres à caractériser les formes sociales inédites, nées du démantèlement des exploitations collectives. Formes familiales et non familiales, petites et grandes exploitations coexistent, en proportions variées, selon les pays. Les profils structurels juxtaposent des formes sociales de production dont les logiques sont de nature différente. La diversité structurelle se caractérise par le gonflement d'un secteur de micro-exploitations, la reconstitution polymorphe d'une

\footnotetext{
* Auteur de correspondance : maurel@ehess.fr
}

agriculture familiale, la reconversion des anciennes fermes étatiques ou collectives en grandes entreprises sociétaires (Bazin et Bourdeau-Lepage, 2011).

Du fait de leur intégration dans l'Union européenne (UE), les nouveaux États membres (NEM) ont été invités à participer à une politique agricole commune (PAC) dont le ciblage se révèle faiblement adapté à la diversité des contextes sociopolitiques et des hétérogénéités structurelles existantes. Devenues bénéficiaires des aides directes et des mesures de soutien de la PAC, les agricultures centre-européennes en expérimentent directement l'impact sur leurs dynamiques de transformation. En fonction du contexte propre à chaque pays, les profils structurels accusent plus ou moins fortement ces effets. Deux aspects des évolutions sont à prendre en compte : les trajectoires de décollectivisation propres aux contextes politiques nationaux, qui expliquent la diversité des profils structurels ; les effets de la PAC qui impliquent l'adoption de 
cadres normatifs et la mise en œuvre de mesures de soutien. Le caractère inédit des transformations à l'œuvre dans ces agricultures constitue un défi pour concevoir et appliquer une politique de ciblage par la catégorisation.

\section{Les limites d'une démarche classificatrice}

Pour identifier les caractéristiques des structures d'exploitation des NEM et en quoi elles se différencient de celles des anciens pays membres (UE-15), on dispose des données statistiques recueillies par Eurostat suivant des méthodologies communes à l'ensemble des États membres de l'UE. Ces catégories statistiques permettent une recension des unités de production agricole selon des critères identiques : la superficie agricole utilisée (SAU), le nombre d'unités de travail annuel (UTA), la dimension économique exprimée en production standard (PS), le statut de l'exploitation, individuelle ou personne morale. Les critères retenus par Farm Structure Survey (FSS) donnent accès à une classification des exploitations agricoles indicative de la singularité structurelle des agricultures des NEM. L'entrée par le niveau de concentration foncière qui privilégie le recours aux critères se rapportant à la taille (SAU) souligne l'existence d'un dualisme agraire marqué par la présence de grandes exploitations et d'un très grand nombre de petites unités de production de moins de 10 ha, avec une forte proportion de micro-exploitations de moins de 2 ha (plus de $70 \%$ du total des exploitations dans les cas de la Hongrie et de la Roumanie). Si l'on répartit la SAU en huit classes selon la taille des unités de production, on constate que dans trois pays, la Slovaquie, la République tchèque et la Bulgarie, plus des quatre cinquièmes de la SAU sont occupés par des exploitations dont la surface est supérieure à 100 ha. L'importance relative de cette catégorie de taille est largement majoritaire en Hongrie, en Estonie, et en Lettonie, proche de la moitié de la SAU totale en Roumanie et en Lituanie, alors qu'elle est particulièrement faible en Pologne et en Slovénie dont les agricultures paysannes avaient échappé à la collectivisation généralisée. Ce premier indicateur statistique ne règle pas la question du seuil de définition de la grande exploitation. Ce seuil doit être apprécié de manière relative dans le contexte de la diversité structurelle propre à chaque pays. En effet, c'est la présence d'exploitations géantes, étendues sur un millier d'hectares et plus, qui distingue les structures agraires d'un certain nombre de NEM. À partir des données de l'enquête FSS, une étude publiée par Eurostat a mis en évidence la place et les traits spécifiques des très grandes exploitations agricoles dont le seuil de délimitation varie relativement à la distribution statistique de l'ensemble des exploitations (Martins et Tosstorff, 2011). La taille moyenne de la très grande exploitation se situe respectivement au-dessus de 2782 ha en Slovaquie, 2500 ha en République tchèque, 1868 ha en Hongrie, 1814 ha en Bulgarie, 1178 ha en Estonie, 832 ha en Roumanie.

Le poids des petites exploitations, en chiffres absolus et relatifs, constitue la deuxième spécificité des structures agraires. Encore faut-il s'entendre sur ce que l'on range dans cette catégorie, en l'absence de définition commune à l'échelle européenne. Une étude publiée par la Commission européenne a appliqué la même méthodologie que celle utilisée pour apprécier les caractéristiques de la très grande exploitation (EU Agricultural Economics Briefs, 2011). Les critères ont trait à la superficie agricole utile, au volume de travail, au niveau de l'autoconsommation, à la taille économique. Le seuil peut être défini en termes absolus ou relatifs (en fonction de la distribution propre à chaque pays). Les agricultures de ces pays se caractérisent par un grand nombre de petites exploitations d'une taille inférieure à 5 ha et souvent à moins de 2 ha. En Bulgarie, Hongrie, Roumanie, les quatre cinquièmes et plus des exploitations recensées ont moins de 5 ha. En Slovaquie, Slovénie, Lituanie, la part relative de cette catégorie dépasse la moitié du nombre total des exploitations. Prises ensemble, ces petites exploitations de moins de 5 ha n'occupent qu'une faible part des terres, généralement inférieure à un dixième de la SAU, à l'exception de la Roumanie où elles forment plus des neuf dixièmes du nombre total sur $28,4 \%$ de la SAU. La comparaison est cependant biaisée du fait qu'en 2010, la République tchèque, la Slovaquie et la Pologne ont relevé le seuil des exploitations suivies par FSS, respectivement à 5,2 et 1 ha, ce qui réduit la couverture de ces catégories.

Cette dualité structurelle s'exprime plus fortement encore en termes de dimension économique (Eurostat, 2014). Les données d'Eurostat soulignent que plus des quatre cinquièmes des unités fournissent moins de 4000 euros de PS en Roumanie et en Bulgarie, et autour de la moitié encore dans les autres pays, à l'exception de la République tchèque. Dans ce dernier pays, ainsi qu'en Slovaquie, les trois quarts de la SAU sont mis en valeur par de grandes exploitations agricoles (plus de 250000 euros).

D'autres modes de catégorisation peuvent être croisés avec l'approche statistique. Les données publiées par Eurostat classent les exploitations en fonction de l'apport en travail de la main-d'œuvre familiale (selon trois niveaux, exclusivement familial, plus de $50 \%$ ou moins de $50 \%$ de l'apport total). L'absence de travail familial devient alors le critère discriminant définissant par défaut une catégorie en marge de l'agriculture dite familiale. La définition large et intégratrice de l'agriculture familiale renvoie à un mode d'organisation associant famille et exploitation (Sourisseau et Even, 2015). Cette catégorie normative englobe un très large éventail de tailles et de situations, de la petite exploitation de semi-subsistance jusqu'à la grande exploitation relevant d'un mode de gestion patronale. Un récent rapport européen analyse la grande diversité de l'agriculture familiale, à laquelle les NEM ne font pas exception (Davidova et Thomson, 2014). La main-d'œuvre familiale apporte une contribution prépondérante en Pologne et en Slovénie (plus des neuf dixièmes du montant des UTA), en Roumanie (plus des quatre cinquièmes), en Lettonie et en Lituanie (plus des trois quarts), et une part majoritaire en Hongrie et en Estonie. Bien qu'en petit nombre, les exploitations non familiales (avec le statut de personne morale) peuvent occuper une place significative en termes de SAU en Estonie (52,2\%), en Hongrie $(48,3 \%)$ et en Roumanie (44\%). Cette forme d'agriculture faisant appel au travail salarié (plus des quatre cinquièmes des UTA) est largement dominante en Slovaquie $(80,7 \%$ de la $\mathrm{SAU})$, en République tchèque $(71 \%)$ et en Bulgarie (60,6 \%). Si les catégories descriptives et normatives soulignent les singularités structurelles de ces agricultures, elles présentent certaines limites. 
Tableau 1. Critères de catégorisation des formes sociales de production.

Table 1. Categorization criteria of production social forms.

\begin{tabular}{|c|c|c|c|c|c|}
\hline $\begin{array}{l}\text { Formes sociales de } \\
\text { production }\end{array}$ & Statut juridique & $\begin{array}{l}\text { Terre (statut du } \\
\text { foncier) }\end{array}$ & $\begin{array}{l}\text { Capital } \\
\text { d'exploitation }\end{array}$ & Travail & Rapport au marché \\
\hline Entreprise sociétaire & $\begin{array}{l}\text { Société par action, } \\
\text { sociétés anonymes, } \\
\text { Sarl }\end{array}$ & $\begin{array}{l}\text { Location majoritaire, } \\
\text { possibilité d'achat } \\
\text { (selon pays) }\end{array}$ & $\begin{array}{l}\text { Parts de capital } \\
\text { (majoritairement } \\
\text { détenues par les } \\
\text { cadres gestionnaires) }\end{array}$ & $\begin{array}{l}\text { Salariat (permanent } \\
\text { et saisonnier) }\end{array}$ & $\begin{array}{l}\text { Orientation marchande } \\
\text { exclusive }\end{array}$ \\
\hline $\begin{array}{l}\text { Exploitation familiale } \\
\text { ou patronale }\end{array}$ & $\begin{array}{l}\text { Exploitation en } \\
\text { responsabilité } \\
\text { individuelle }\end{array}$ & $\begin{array}{l}\text { Faire-valoir direct et } \\
\text { indirect }\end{array}$ & $\begin{array}{l}\text { Bâtiments, équipement } \\
\text { productif, cheptel, etc. }\end{array}$ & $\begin{array}{l}\text { Principalement } \\
\text { familial }\end{array}$ & $\begin{array}{l}\text { Orientation marchande } \\
\text { dominante }\end{array}$ \\
\hline $\begin{array}{l}\text { Petite exploitation de } \\
\text { subsistance ou de } \\
\text { semi-subsistance }\end{array}$ & Non enregistrée & Faire-valoir direct & $\begin{array}{l}\text { Petit matériel, } \\
\text { quelques têtes de } \\
\text { bétail }\end{array}$ & $\begin{array}{l}\text { Exclusivement } \\
\text { familial }\end{array}$ & Orientation vivrière \\
\hline
\end{tabular}

Source : l'auteur.

\section{En l'absence de référentiel, une catégorisation des formes sociales par leur filiation}

L'analyse des agricultures qui ont connu le collectivisme agraire, selon des formes et des modalités variables, requiert une démarche spécifique. Pour qualifier des formes sociales originales, on propose d'introduire une catégorisation à visée interprétative. Cette démarche part de la transformation des profils structurels de ces agricultures, déclenchée par la rupture avec le système d'économie socialiste. Une vision libérale de l'économie de marché, fondée sur la reconnaissance de la propriété privée et de la liberté d'entreprendre, a présidé au changement de système. La remise en cause des fondements mêmes du collectivisme s'est opérée sans que la question d'un modèle agricole de substitution soit posée. Faute de consensus politique entre des groupes d'acteurs aux intérêts divergents, les bouleversements agraires se sont accomplis en l'absence de référentiel politique cohérent susceptible d'orienter la mutation vers un autre modèle agricole (Maurel, 1994). Priorité a été donnée par le législateur à l'établissement des droits de propriété sur la terre et le capital d'exploitation, ainsi qu'au changement de statut des anciennes exploitations collectives. Dans certains cas (en Roumanie), un processus spontané de démantèlement des exploitations collectives a pu précéder l'application d'un dispositif encadrant la transformation. Dans le contexte de la transition de système, libre cours a été laissé aux initiatives des acteurs sociaux les plus entreprenants pour se saisir des opportunités d'accession à la terre et au capital de production tandis que les modalités de conversion des structures collectivistes ont été définies sans réelle considération des conditions de viabilité des exploitations successeurs. Les dispositions encadrant le processus de décollectivisation ont eu pour effet de dissocier les trois facteurs de production que sont la terre, le capital et le travail, auparavant assemblés dans le cadre des exploitations collectives. Très complexes dans leur conception comme dans leurs modalités d'application, les dispositifs ont combiné le rétablissement des droits de propriété antérieurs à la collectivisation avec l'attribution de parcelles de terre aux travailleurs agricoles (en Hongrie) ou aux ménages ruraux (en Lituanie). De manière générale, la reconnaissance des droits de très nombreux petits propriétaires a déterminé une extrême fragmentation du capital foncier tandis que la recomposition du capital d'exploitation dans les mains de nouveaux acteurs autonomes a donné naissance à des formes sociales inédites. Une profonde transformation des rapports fonciers et des rapports de production s'en est suivie. La diversité des formes sociales résulte de la déconcentration foncière et de la mutation des modes d'organisation du travail agricole.

Pour qualifier et décrire ces formes sociales, il convient de tenir compte de l'affectation des droits de propriété sur la terre et sur le capital d'exploitation, ainsi que du mode d'articulation des rapports de propriété et des rapports de production au sein des entités ainsi transformées. Cette approche s'applique aux exploitations qui ont pris la succession des structures collectivistes (coopératives de propriétaires, associations agricoles, sociétés de divers types, etc.) comme aux exploitations nouvellement installées. Ce mode de catégorisation à visée interprétative concerne des formes sociales de production aux configurations fort diverses. Les modalités plurielles prises par la recomposition des facteurs de production rendent improbable la différenciation en fonction des critères habituellement mobilisés (l'organisation du travail, les logiques patrimoniales et productives, les stratégies de développement, etc.). À cette difficulté, s'ajoute le fait que certaines formes sociales, nées d'une combinaison hybride des facteurs de production, se révèlent transitoires. Après quelques années, les néocoopératives de propriétaires, les entreprises publiques (issues de la conversion des fermes d'État) ont transformé leur statut juridique de manière à stabiliser le capital d'exploitation et à consolider le mode de gestion managériale. Par ailleurs, les nouvelles exploitations de type familial, établies à la suite de la privatisation des terres, se sont révélées vulnérables et ont pu disparaître. Les recompositions procédant de la déconcentration foncière et de la mutation des modes d'organisation du travail agricole se sont déployées dans le contexte de politiques nationales qui ont beaucoup fluctué, notamment en termes de ciblage d'une catégorie par rapport aux autres. À partir de nos enquêtes de terrain 
conduites sur plus de deux décennies, chaque forme sociale de production peut ainsi être définie par un mode d'articulation de la terre, du capital et du travail qui lui est spécifique (Tab. 1). Leur filiation relève de mécanismes complexes de transmission, de reproduction et d'adaptation.

\subsection{Une domination des grandes exploitations sociétaires}

Elles sont issues de la privatisation des fermes d'État et de la conversion des coopératives de propriétaires. Généralement privatisées à l'initiative de leurs anciens cadres dirigeants, dans le cadre de stratégies d'appropriationcontrôle de leur capital, elles fonctionnent sous une forme sociétaire (société par actions, société à responsabilité limitée), emploient des salariés et produisent exclusivement pour le marché (Maurel, 2012). Ces grandes exploitations dont la taille moyenne est de l'ordre de quelques centaines à quelques milliers d'hectares cultivent une part prépondérante de la SAU en Slovaquie, en République tchèque, en Bulgarie et en Estonie, et plus des deux cinquièmes en Hongrie et en Roumanie. Par leur haut niveau capitalistique, leur intégration au sein de grands groupes agro-alimentaires, une partie de ces entreprises participent de l'agriculture de firme (Purseigle, 2012).

\subsection{Une réapparition polymorphe de l'agriculture familiale}

L'opportunité de retirer des terres et des équipements des anciennes exploitations collectives, et la possibilité de bénéficier des restitutions ont permis à des agriculteurs de s'installer de manière indépendante. La réappropriation d'un bien foncier par une famille ne suffit pas à garantir la viabilité $\mathrm{du}$ projet d'installation dans les conditions incertaines qui ont présidé à la sortie du collectivisme (Maurel, 2015). Cette agriculture familiale revêt diverses formes sociales qui se distinguent par les dimensions, la logique de fonctionnement, l'orientation de la production vers le marché et/ou l'autoconsommation. On la distinguera de la petite agriculture de semi-subsistance.

Renouant avec une logique familiale, fondée sur une articulation étroite entre le capital et le projet familial, les exploitations installées au lendemain de la décollectivisation associent faire-valoir direct et indirect. Les difficultés rencontrées pour accéder au crédit, bénéficier d'une assistance technique adéquate et écouler la production sur le marché expliquent la faible emprise de cette forme d'exploitation familiale. L'évolution récente se caractérise par le recul du nombre d'exploitations tandis que s'opère une lente consolidation d'exploitations moyennes familiales ou patronales dans plusieurs pays (Lituanie, Lettonie, Hongrie). Seules les unités relevant des classes au-delà de 20 ha, et surtout de 50 ha, se trouvent en situation d'élargissement foncier, d'investissement et de modernisation. Les hétérogénéités structurelles s'accentuent. Une agriculture marchande fonctionnant dans un modèle de type entreprise familiale, engagée dans des stratégies d'accu- mulation foncière, constitue le pôle moderniste. Dans leur grande majorité, ces exploitants cherchent à élargir leur assise foncière mais l'insuffisante fluidité du marché foncier constitue une entrave à leur développement. La consolidation de cette agriculture professionnelle impliquerait une capacité d'accumulation que leur compétitivité n'autorise pas. En l'absence de ciblage des aides à l'investissement en faveur de ces exploitations, la mutation moderniste sur un modèle marchand reste un processus hautement sélectif.

\subsection{Une myriade de petites exploitations de semi-subsistance}

Les plus petites exploitations ont pour origine les anciens lopins de l'économie auxiliaire attribués aux travailleurs agricoles pour leur usage propre (autoconsommation). Les politiques de redistribution des terres ont permis d'agrandir ces lopins (en Lituanie jusqu'à 3 ha). Les critères de définition de ces micro-exploitations (superficie, taille économique, participation au marché) varient selon les pays. Leur statut juridique est souvent mal établi en raison de l'absence d'enregistrement de leur activité productive. La logique productive est dictée par la satisfaction des besoins alimentaires du ménage. Ces exploitations assurent la survie des populations rurales les plus démunies (retraités, chômeurs). Lors de la crise de transition, ces petites unités de production ont joué un rôle décisif d'amortisseur social. En fournissant un complément de revenu aux pluriactifs comme aux agriculteurs âgés, elles remplissent une fonction sociale qui contribue à alléger la pauvreté en milieu rural. Le vieillissement de cette catégorie d'exploitants offre des perspectives pour les changements structurels (notamment en Hongrie) (Vandenbroucke et Fehér, 2011). Une étude concernant l'agriculture de semi-subsistance répertorie ces exploitations en sous-groupes, selon leur taille (moins de 2 ha, moins de 5 ha) et leur PS (inférieure à 2000 euros, inférieure à 8000 euros) (Davidova et al., 2013). Leur importance relative en nombre d'exploitations est considérable en Roumanie, en Hongrie, en Lettonie, sensiblement moindre en Slovénie, Lituanie, Bulgarie, Pologne et Estonie, et marginale en République tchèque.

Les itinéraires de recomposition des structures agraires ont emprunté des trajectoires plurielles (Halamska et Maurel, 2010). Les profils structurels juxtaposent des formes sociales dont les filiations et les logiques sont différenciées. Ainsi, peuvent être distinguées des agricultures à forte concentration foncière et à base de travail salarié (République tchèque, Slovaquie, Estonie), des agricultures duales composées de petites et de grandes exploitations (Hongrie, Roumanie, Bulgarie, Lettonie), et des agricultures à dominante de petites et moyennes exploitations familiales (Lituanie, Pologne, Slovénie).

\section{Les effets structurels de la PAC: un ciblage inapproprié}

Les pays d'Europe centrale ont éprouvé des difficultés pour construire leur propre référentiel de politique agricole. À certains moments, les politiques nationales ont pu 
soutenir la constitution d'exploitations de type familial (notamment en Lituanie), mais les décideurs politiques ont pu aussi favoriser les grandes exploitations sociétaires, proches de l'agro-business (en Hongrie). Dans un contexte de relative instabilité de la scène gouvernementale, ces changements de cap sont l'expression de l'inégale capacité d'intervention de groupes d'intérêts hétérogènes.

L'entrée dans l'UE (2004-2007) et la mise en œuvre de la PAC ont eu un impact évident sur les évolutions structurelles. L'introduction progressive de mesures de soutien destinées à encourager la restructuration dans une perspective de convergence avec les anciens États membres s'est opérée sans que l'on s'interroge sur leur adéquation au vu des contextes propres à ces agricultures. Or, le référentiel politique de l'agriculture familiale qualifiée de «modèle opérationnel dominant en Europe " s'est révélé largement inapproprié (Swain, 2013). Dans le cadre limité de cette étude, on se borne à évoquer les choix de ciblage des piliers de la PAC. Les effets de ces mesures sur les évolutions observées sont de deux ordres, direct et indirect.

Mises en application aux marchés et aux revenus dans le cadre du régime simplifié de paiements directs, les mesures de soutien agricoles du premier pilier procèdent d'une approche orientée sur les grandes exploitations agricoles. Les ajustements opérés en faveur des plus petites exploitations ont permis d'abaisser le seuil de la superficie éligible et d'augmenter le montant attribué. En dépit du relatif élargissement du nombre de bénéficiaires, l'aide directe versée aux petits exploitants, compte tenu des taux de paiement à l'hectare, ne constitue pas une solution pour les petites exploitations. Les nombreux bénéficiaires ne perçoivent qu'une très faible part des paiements directs et leur versement est assujetti au respect d'exigences minimales. Les mesures du second pilier, dédié au développement rural, ne sont pas plus favorables aux petites exploitations. Les exploitations de semisubsistance bénéficient d'un dispositif d'aide à la restructuration (la mesure 141) qui relève de la politique de développement rural adoptée par certains de ces pays (Bulgarie, Hongrie, Lettonie, Lituanie, Roumanie). Quant aux exploitations moyennes, elles reçoivent des aides directes modestes qui n'autorisent pas des investissements significatifs. D'autres mesures qui dépendent du second pilier de la PAC peuvent soutenir la modernisation des exploitations (mesure 121), à partir d'un seuil d'éligibilité variable selon les pays, mais les conditions d'accès sont très sélectives et découragent les exploitants susceptibles d'en bénéficier. De ce fait, leur consolidation demeure lente. Les grandes exploitations sociétaires sont les principales bénéficiaires du régime simplifié des paiements directs devenus l'élément moteur de la concentration foncière.

La situation foncière est paradoxale : l'extrême fragmentation de la propriété foncière ne se traduit pas dans la trame d'usage des terres caractérisée par une relative concentration au profit des grandes exploitations, sous la forme de baux de location. Une proportion dominante des terres se trouve en faire-valoir indirect. Les formes de la concentration foncière sont diverses en fonction des caractéristiques du marché de la terre. Longtemps resté peu animé, le marché foncier est à présent soumis à des évolutions rapides du fait de la fin du moratoire interdisant l'achat des terres par les étrangers. Dans la plupart de ces pays, la pénétration des investisseurs européens accélère le processus d'accaparement foncier au bénéfice des très grandes exploitations et la marginalisation des petites et moyennes structures.

\section{Conclusion}

Quelques tendances majeures se dessinent. Les effets distributifs de la PAC jouent en faveur de la seule catégorie des grandes exploitations en marginalisant les autres formes sociales de production. Loin de contribuer au développement d'une agriculture de type familial, la PAC a eu pour effet de renforcer la position des grandes structures issues de la transformation des exploitations collectives tandis qu'elle rendait improbable la consolidation d'exploitations familiales de dimensions viables. Le processus de concentration au profit des grandes exploitations progresse aux dépens des plus petites dont le nombre recule fortement tandis que les exploitations de type familial, mal établies et incomplètement consolidées, demeurent de ce fait vulnérables. Le ciblage par la catégorisation tel que, par exemple, le soutien des exploitations de semisubsistance semble inopérant. Le référentiel du modèle agricole européen s'est révélé largement inapproprié à la réalité socio-économique des agricultures centre-européennes et ses effets pervers peuvent être soulignés. À l'opposé de la convergence attendue, une forte hétérogénéité structurelle caractérise la plupart des agricultures centre-européennes. À quelques exceptions près (Pologne, Slovénie, Lituanie), la marque d'un dualisme agraire de longue durée s'est à nouveau imposée.

\section{Références}

Bazin G, Bourdeau-Lepage L. 2011. L'agriculture dans les pays d'Europe centrale et orientale : continuité et adaptation. Econ. Rural. 325-326: 10-24.

Davidova S, Thomson K. 2014. Family farming in Europe: challenges and prospects. Brussels: DG for Internal policies, Policy department B: Structural and cohesion policies, $59 \mathrm{p}$.

Davidova S, Bailey A, Dwyer J, Erjavec E, Gorton M, Thomson K. 2013. Semi-subsistence farming: values and directions of development. Brussels: DG for Internal policies, Policy department B: Structural and cohesion policies, $112 \mathrm{p}$.

EU Agricultural Economics Briefs. 2011. What is a small farm? Brief $\mathrm{n}^{\mathrm{o}}$ 12. Brussels: EU Agricultural Economics Briefs.

Eurostat. 2014. Agriculture, forestry and fishery statistics. Brussels: Eurostat. Disponible sur http://ec.europa.eu/eurostat/statisticsexplained/index.php/.

Farm Structure Survey (FSS).

Halamska M, Maurel M-C. 2010. Decollectivization of agriculture and reshaping of agrarian structure in Central Europe. Prz. Socjol. 59(2): 29-56.

Martins C, Tosstorff G. 2011. Large Farms in Europe.Statistics in focus: agriculture and fisheries. Report $\mathrm{n}^{\circ} 18$. Brussels: Eurostat.

Maurel M-C. 1994. La transition postcollectiviste : mutations agraires en Europe centrale. Paris: L'Harmattan.

Maurel M-C. 2012. La grande maille agraire en Europe centrale : un invariant spatio-temporel ? Etudes Rural. 190: 25-48. 
Maurel M-C. 2015. From disappearance to recovery: family farming in Central Europe. Questioning the path dependency hypothesis. Wies Rol. Warsz. 166(1): 79-105.

Purseigle F. (coord.). 2012. Les agricultures de firme. Organisations et financiarisation. Etudes Rural. 1(190): 208 p.

Swain N. 2013. Agriculture 'East of the Elbe' and the Common Agricultural Policy. Sociol. Rural. 53(3): 369-389.
Sourisseau J-M, Even M-A. 2015. Transformations agricoles et agricultures familiales: quelques défis mis en lumière durant l'Année internationale de l'agriculture familiale. Cah. Agric. 24: 201-203, doi: 10.1684/agr.2015.0764.

Vandenbroucke P, Fehér I. 2011. Dynamique des structures agraires hongroises : quels profils d'exploitations se dessinent? Econ. Rural. 325-326: 100-113

Citation de l'article : Maurel M-C. 2016. Penser la catégorisation des formes sociales de production agricole en Europe centrale. Cah. Agric. 25: 45002 . 